\title{
A comparative study of rebamipide $2 \%$ and carboxy methylcellulose (CMC) $0.5 \%$ eye drop in patients with dry eyes: A randomized, hospital-based study
}

\author{
Dipak B. Patel ${ }^{1}$, Palvee Menon ${ }^{2, *}$, Anshi Rathod ${ }^{3}$, Aneesha Vyas ${ }^{4}$, Poonam Rana $^{5}$ \\ ${ }^{\mathbf{1}}$ Professor, ${ }^{\mathbf{2 , 3}, \mathbf{4}}$ Resident, Dept. of Ophthalmology, SBKS Medical Institute \& Research Center, Sumandeep Vidyapeeth, \\ Vadodara, Gujarat, ${ }^{\mathbf{5}}$ Consultant, Supreme Hospital Eros Garden, Faridabad, Haryana, India
}

*Corresponding Author: Palvee Menon

Email: palveemenon@gmail.com

\begin{abstract}
Aim: To compare the efficacy of $2 \%$ rebamipide and $0.5 \%$ Carboxy methylcellulose (CMC) eye drops in dry eye patients. Materials and Methods: A 100 dry eye patients were enlisted and screened. Patients were allotted randomly into 2 groups(Group-A) 2\% rebamipide or (Group-B) 0.5\% Carboxy methylcellulose (CMC) ophthalmic solution, after screening and instilled as 1 drop in each eye four times a day, for about two months. The signs and symptoms were assessed -at baseline, 2, 4, and at 8week visits. The objective signs were -"Schirmer test and Tear film break-up time" (TBUT) while subjective symptoms were dry eye related ocular symptoms (foreign body sensation, dryness, photophobia, eye pain, and blurred vision.

Results: A compelling improvement was noticed in the Schirmer test and TBUT values after the treatment in the $2 \%$ rebamipide group. The patients on $2 \%$ rebamipide eye drop showed considerable improvement in dry eye related ocular symptoms, than the patients on $0.5 \%$ CMC eye drop (51\% and $29 \%$ respectively) at 2 months.

Conclusions: $2 \%$ rebamipide ophthalmic solution was more efficient in improving both the subjective symptoms and objective signs of dry eye in comparison to the CMC group in this 8 -week study. These findings showed that $2 \%$ rebamipide is the more effective drug for dry eye.
\end{abstract}

Keywords: Carboxy methylcellulose, Dry eye, Efficacy, Rebamipide.

\section{Introduction}

Dry eye disease is a disorder of tear film causing ocular symptoms like foreign body sensation, dryness, blurring of vision, photophobia, and tear-film dysfunction. The "prevalence" of dry eye in India was accounted to be $29.25 \% .^{1}$ The primary causative reasons of dry eye are lack of tear production or increased tear evaporation, which causes deterioration of the ocular surface and is associated with symptoms of ocular discomfort. It effect the tear film osmotic concentration and causes ocular surface inflammation. ${ }^{2}$ Dry eye disease occurs mostly in patients with deteriorating general health and various systemic conditions, including arthritis, fractures, osteoporosis, gout, and thyroid disorders. It is also related with increasing age, history of allergy, diabetes mellitus, connective tissue disease, use of antihistamines drugs and refractive surgery. ${ }^{3-7}$

Rebamipide is a mucin stimulator drug. It is a unique quinolinone by-prouduct. It helps the gastric mucosa by increasing the prostaglandins, and develops gastric epithelial mucin. It helps in cytoprotection, healing of wound, anti-inflammatory properties and free radical scavenger. It's has been vastly used for gastritis and gastric ulcers, since $1994 .{ }^{8}$ After ascertaining it's pharmacological actions, it began its development to be used as a topical drug in the dry eye patients. It has been noted that rebamipide rises mucin production of cornea and conjunctiva, and goblet cells of conjunctiva and also rises the mucin production in cultured goblet cells of conjunctiva and in epithelial cells of cornea. These reports, in addition, indicated that rebamipide augment the secretion of mucins. ${ }^{10}$

Carboxy methylcellulose (CMC) is the most frequently used drug as a artificial drops in dry eyes. CMC prolongs the retention time, duration of activity, lubrication, and hydration of the ocular surface. Both the drugs are approved (DCGI) and routinely used in the treatment of dry eye. Minor side effects with CMC like burning, stinging, irritation and rarely allergic reactions like rash, itching, swelling, and dizziness have been reported.

Rebamipide have side effects of bad taste (dysgeusia) and very rarely have a stinging effect. The current study was conducted with an aim to assess the effectiveness of $2 \%$ rebamipide and $0.5 \%$ CMC eye drops in the dry eye.

\section{Materials and Methods}

This is a randomized interventional prospective study. It was conducted on 100 dry eye patients in the Ophthalmology Department, Dhiraj Hospital, Piparia from Jun 2017 to December 2017. Patients were enlisted after procuring a permission from the Ethical Committee and after obtaining the informed written consent. Subjects with age above 18 years and of both sexes who fit in inclusion criteria were taken in the study. The inclusion criteria are as follows: a) A score of $>2$ for 1 or more dry eye-related ocular symptoms b) An Schirmer's II test value at five minutes of less than $5 \mathrm{~mm}$ or TBUT less than 5 seconds c) Best corrected visual acuity (BCVA) more than 6/36. Patients who had tear film dysfunction, blepharitis, blepharospasm, 
glaucoma, uveitis, punctal plugs, vascular disease, history of ocular surgery within one year and history of any ocular medications which causes dry eye were excluded. After randomly allocated the patients into 2 groups, so (Group-A 2\% rebamipide and Group-B 0.5\% CMC) are formed. Fifty subjects were in each group who received either $2 \%$ rebamipide or $0.5 \%$ CMC. Patients were advised to administer 1 drop in each eye for 4 times in a day for 2 months. The following parameters were evaluated like complete visual acuity, tear film function by tear film break-up time (TBUT), and tear secretion (Schirmer's I, Schirmer's II), and slit lamp examination of the height of tear meniscus(TMH) at each visit. TMH value was evaluated by measuring the height of the lacrimal river and reading the scale on slit lamp microscopy.

\section{Statistical Analysis}

All the results are expressed as the number, percentages and mean \pm standard deviation (SD). The comparison of continuous variables between the groups was performed by $\mathrm{Z}$ test. A value of $\mathrm{p}$ less than 0.05 is considered significant.

\section{Result}

A total of 100 dry eye patients (200 eyes) enlisted in the study. Study blinding was throughout maintained. The response of $2 \%$ rebamipide eye drops and $0.5 \%$ CMC was observed at baseline (day of $1^{\text {st }}$ visit), 2 weeks, 4 weeks and 8 weeks. Of the 100 patients, 65 were male. The mean age was 29.8 years (range $18-54$ years).

Schirmer Test: In right eye, the mean baseline Schirmer I scores was 7.8 in the both Groups. The mean scores at 2 weeks (13.4 in both groups), 4 weeks (15.7 and 13.6 respectively) and 8 weeks (19.5 and 15.0 respectively) (Fig. 1). The mean baseline Schirmer II scores in right eye were 6.0 in both Groups. The mean scores at 2 weeks (11.7 in both group), 4 weeks (13.7 and 12.4 respectively) and 8 weeks (17.0 and 15.3 respectively) (Fig. 2). A significant difference in Schirmer I and Schirmer II between rebamipide 2\% and CMC groups were observed at 4 weeks and 8 weeks. In the left eye similar pattern was reported.

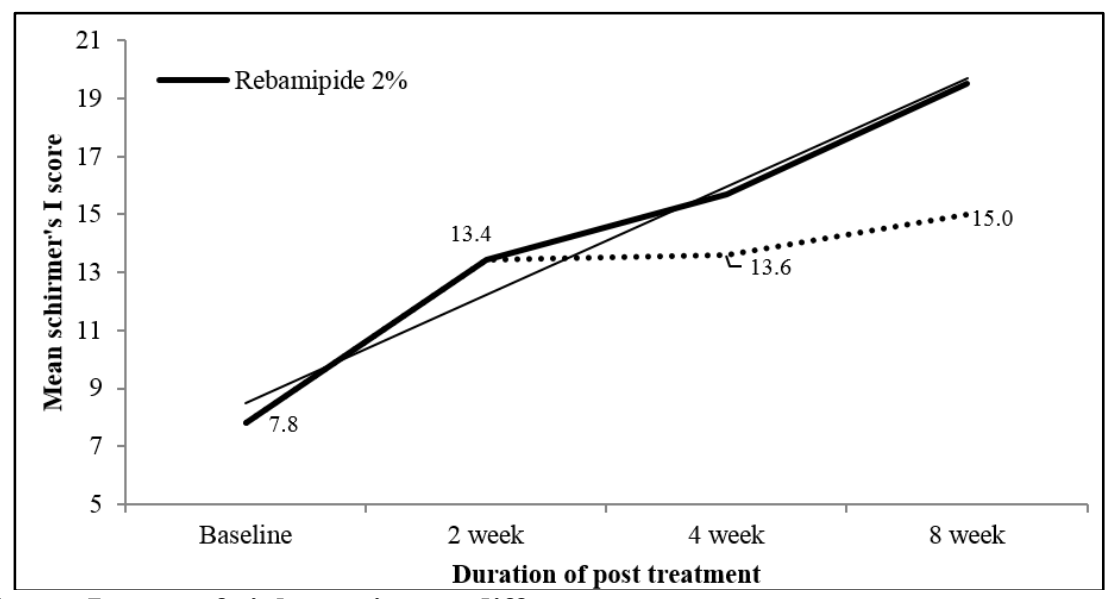

Fig. 1: Mean Schirmer I score of right eye in two different treatment groups

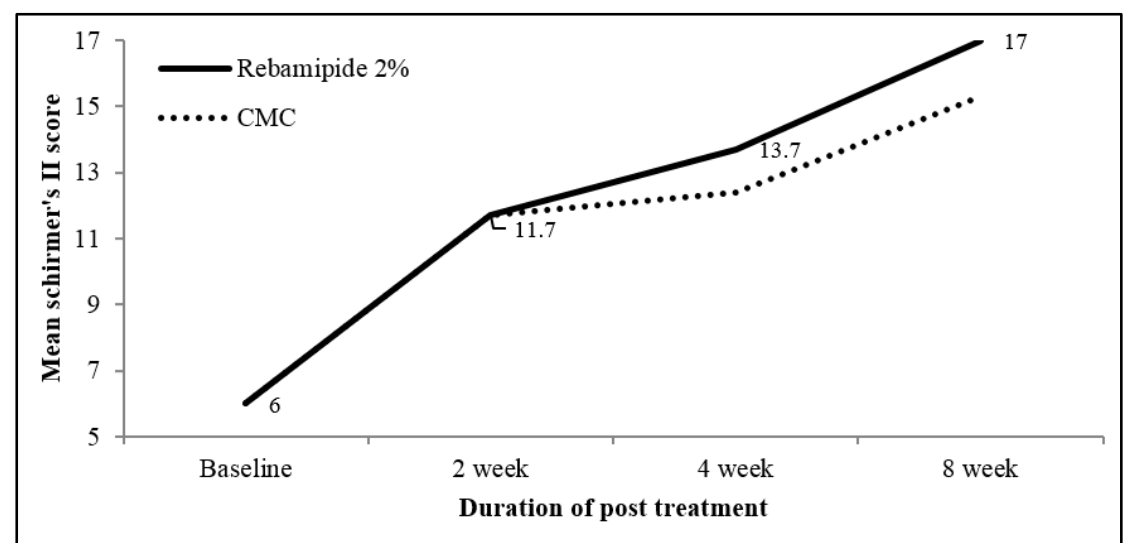

Fig. 2: Mean Schirmer II score of Right eye in two different treatment groups

Tear Film Break-Up Time: The mean TBUT values in the right eye at baseline were 7.3 and 7.4 in rebamipide and CMC groups, respectively. At 2 week, mean TBUT in both groups was similar (7.6). There was a 
significant difference $(\mathrm{p}<0.05)$ between the mean scores was observed at 4 weeks (8.5 and 7.2 respectively) and 8 weeks (12.2 and 7.8 respectively) (Fig. 3). A similar pattern was observed in the left eye. The mean change from baseline in TBUT in the right eye was 4.9 and 0.5 in the $2 \%$ rebamipide and CMC groups, respectively.

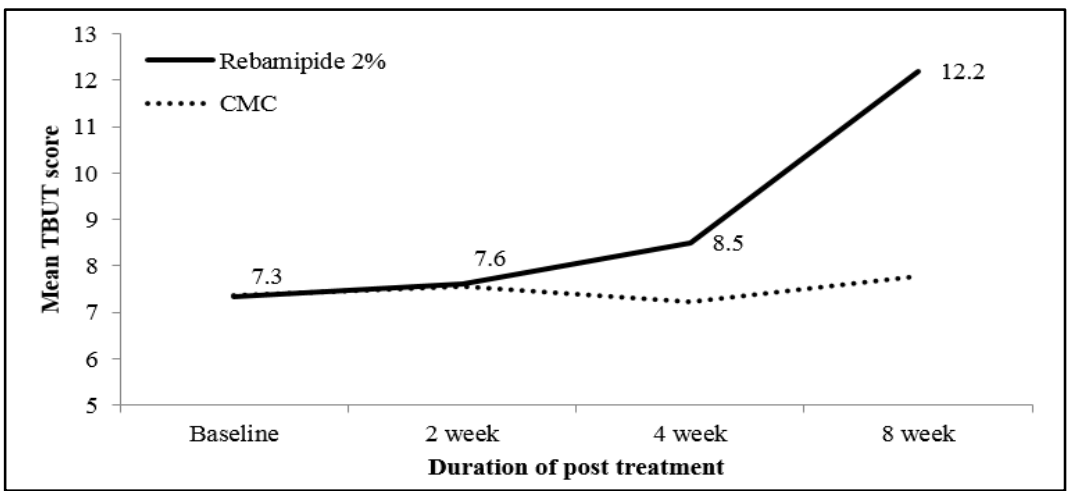

Fig. 3: Mean TBUT score of right eye in two different treatment groups

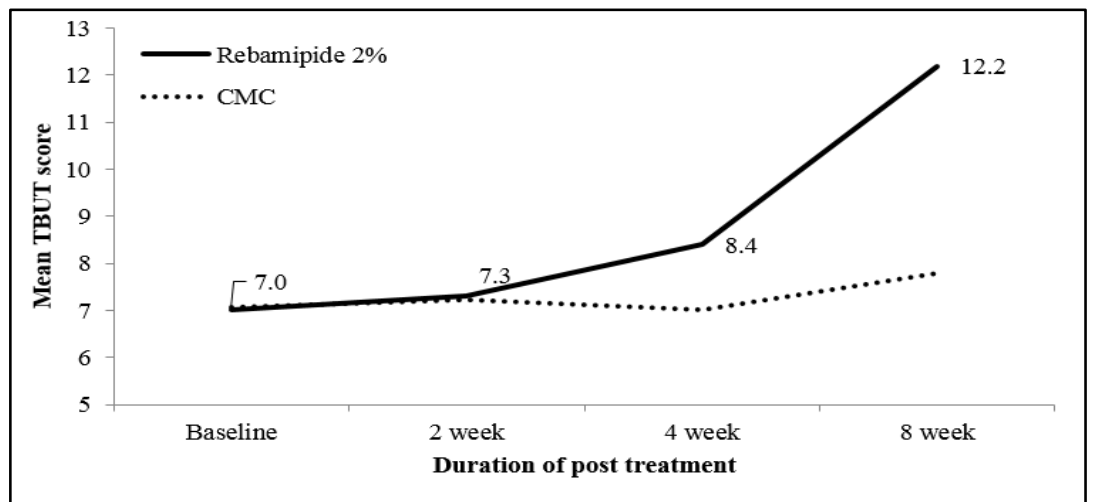

Fig. 4: Mean TBUT score of left eye in two different treatment groups

At baseline mean dry eye-related ocular symptom scores were same in both treatment groups (foreign body sensation: 2 and 2; dryness: 3 and 3; photophobia: 2 and 2; eye pain: 2 and 2; and blurred vision: 2 and 3 in $2 \%$ rebamipide group and $0.5 \% \mathrm{CMC}$ group respectively). All the 5 dry eye-related ocular symptom scores were significantly improved in the $2 \%$ rebamipide group in contrast to $0.5 \% \mathrm{CMC}$ group at 4 and 8 weeks. A significant change from baseline was seen.

Adverse effects were reported in 13 patients (26.0\%) receiving $0.5 \% \mathrm{CMC}$, and 11 patients $(22.0 \%)$ receiving $2 \%$ rebamipide. Eye irritation and Dysgeusia were observed as adverse effects.

\section{Discussion}

In our study, patients on rebamipide eye drop are improved significantly in relation to the signs and symptoms of dry eye.

A latest study additionally showing that rebamipide augment mucin-like glycoprotein and MUC1 and MUC4 gene expression when human corneal epithelial cells were nurture with rebamipide ${ }^{11}$ Similarly, a clinical study was conducted in Japan showed that the attenuation of the giant papillae in all the 4-VKC patients contrary to routine anti-allergic treatment. ${ }^{12}$ similarly, S Kinoshita observed significant difference at 4 weeks between rebamipide $2 \%$ and placebo groups. ${ }^{13}$

In our study, a significant difference of mean Schirmer's and TBUT score between 2\% rebamipide and $0.5 \%$ CMC groups was observed at 4 and 8 weeks. It suggests that rebamipide $2 \%$ eye drops improves the tear production and quality in dry eye patients by increasing the mucin production from the goblet cells.

The reported adverse effects were similar between the 2 groups. Significantly, dysgeusia was the more commonly observed adverse effect within the rebamipide group. However, there are few constraints to the current study because the sample size was too small and follow up was short.

\section{Conclusion}

In the treatment of dry eye disease, the $2 \%$ rebamipide was more effective and better tolerated. There was a statistically vital improvement in patient's overall treatment impressions in $2 \%$ rebamipide group as compared with the $0.5 \%$ CMC group (51\% and $29 \%$, respectively). Such improvements should contribute to improved quality of life in patients with dry eye. 


\section{References}

1. Mertzanis P, Abetz L, Rajagopalan K, Espindle D, Chalmers R. The relative burden of dry eye in patients' lives: comparisons to a U.S. normative sample. Invest Ophthalmol Vis Sci. 2005;46(1):46-50.

2. Lemp MA, Baudouin C, Baum J. The definition and classification of dry eye disease: report of the definition and classification subcommittee of the international dry eye workshop. Ocul Surf. 2007;5(2):75-92.

3. Moss SE, Klein R, Klein BE. Incidence of dry eye in an older population. Arch Ophthalmol. 2004;122(3):369-73.

4. Moss SE, Klein R, Klein BE. Long-term incidence of dry eye in an older population. Optom Vis Sci. 2008;85(8):668-74.

5. De Paiva CS, Chen Z, Koch DD, Hamill MB, Manuel FK. The incidence and risk factors for developing dry eye after myopic LASIK. Am J Ophthalmol. 2006;141(3):438-45.

6. Shoja MR, Besharati MR. Dry eye after LASIK for myopia: Incidence and risk factors. Eur J Ophthalmol. 2007;17(1):1-6.

7. Schaumberg DA, Dana R, Buring JE, Sullivan DA. Prevalence of dry eye disease among US men: Estimates from the physician's health studies. Arch Ophthalmol. 2009;127(6):763-8.

8. Gupta N, Prasad I, Jain R, D'Souza P. Estimating the prevalence of dry eye among Indian patients attending a tertiary ophthalmology clinic. Ann Trop Med Parasitol. 2010;104(3):247-55.

9. Logaraj M, Madhupriya V, Hegde S. Computer syndrome and Associated factors Among Medical and Engineering students in Chennai. Ann Med Health Sci Res. 2014;4(2):179-85.
10. Kashima T, Itaura H, Akiyama H, Kishi S. Rebamipide ophthalmic suspension for the treatment of dry eye syndrome: A critical appraisal. Clinical Ophthalmology. 2014;8:1003-1010.

11. Takeji Y, Urashima H, Aoki A, Shinohara H. Rebamipide increases the mucin-like glycoprotein production in corneal epithelial cells. J Ocul Pharmacol Ther. 2012;28(3):259-63.

12. Ueta M, Sotozono C, Koga A, Yokoi N, Kinoshita S. Usefulness of a new therapy using Rebamipideeyedrops in patients with $\mathrm{VKC} / \mathrm{AKC}$ refractory to conventional anti-allergic treatments. Allergologyinternational. 2014;63(1):75-81.

13. Kinoshita S, Awamura S, Oshiden K, Nakamichi N, Suzuki H, Yokoi N. Rebamipide (OPC-12759) in the Treatment of Dry Eye: A Randomized, Double-Masked, Multicenter, Placebo-Controlled Phase II Study. Ophthalmology. 2012;119(12): 2471-8.

How to cite this article: Patel D.B, Menon $\mathrm{P}$, Rathod A, Vyas A, Rana P. A comparative study of rebamipide $2 \%$ and carboxy methylcellulose (CMC) $0.5 \%$ eye drop in patients with dry eyes: A randomized, hospital-based study. Indian J Clin Exp Ophthalmol. 2018;4(4):526-529. 